\title{
Lukewarm and Trembling Therapy Device to Assist the Reduction of Blood Flow for Immobilized Patients
}

\author{
Malene Helgo \\ Federal University of Maranhão, Vila Bacanga, Sao Luís - MA, 65080-805, Brazil \\ malenehelgo91@aol.com
}

\begin{abstract}
Article Info
Journal of Computing and Natural Science (http://anapub.co.ke/journals/jens/jens.html)

Doi: https://doi.org/10.53759/181X/JCNS202101004

Received 26 October 2020; Revised form 26 November 2020; Accepted 28 December 2020.

Available online 05 January 2021.

(C)2021 Published by AnaPub Publications.
\end{abstract}

\begin{abstract}
Reduction of blood flow due to immobility of legs de-escalates transportability of liberal number of patients in a extensive range of healing scheme transverse from bruise grume. In normal human being effective system of venous rejoin both alive and yielding is amenable for prohibition of profound venous apoplexy. Ultrasonic blood flow rate sensor, Tilt sensor, Force sensor and Accelerometer sensor are used to detect and identify the flow rate as well as ankle position. The paper offers prophylaxis and slimnastics device that caricature the essential proposition off venous recompense to uphold the blood flow gadget is based on Lukewarm and Trembling technique that is compress and appropriately from equipped in pattern and additionally requirement no restricted trainings for the operation.
\end{abstract}

Keywords - Prophylaxis and Slimnastics, Lower Limb, Blood Flow, Assistive Technology.

\section{INTRODUCTION}

The protracted immobilization of legs is either owing to an extremely long-time alleviated stance of long suffering in a operation theatre or account of age confined bed baited patient spread develops apoplexy in the deep vein. The problem discloses as blister in the legs which can consequence in a secondary epidemiology. Depending on the magnitude of occlusion, various indicator and ill health process are instigated [1]. This circumstance in sustained aspect can result in varicose ulcers in the leg which are wound healing. The patients who are incapacitated, leg motility is degrade and this conviction decline in the flow of blood within the deep veins. A wide range of patients sustain from seizures related effects. Patients who have experienced biopsy, orthopedic plane of action, suffer rupture in the lower limb; lack of neuromuscular deficiency are all under this group of disease. Two distinct perspective are implemented. One has been pharmaceutic fashion of using purgative intoxicants. Another approach is non-pharmaceutic type includes involuntary collapse. Thus, pharmacological means have the highest risk; whereas machine-like methods are relatively safe have lower efficiency in avert thrombosis. In this paper, a pattern of a device to abet the reduction of blood flow has been proposed. To enlarge the blood flow in the lower limb, here we use Lukewarm and trembling therapy for immobilized patients. Flow of blood in the deep venous system under rest state and inflated states are shown in Fig 1.

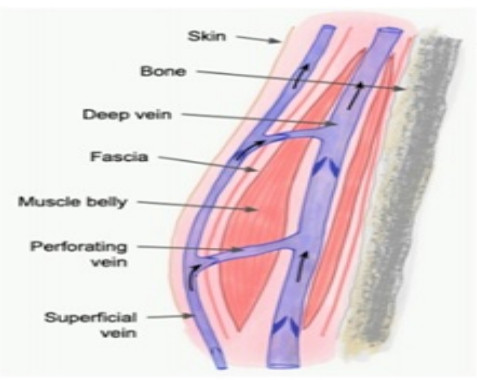

(a)

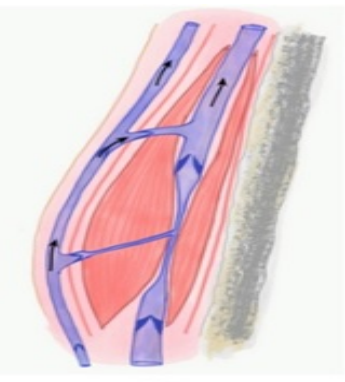

(b)

Fig 1. Flow of blood in the deep venous system under (a) rest state, and (b) under inflated state.

The working prototype has been evolved and appealed on healthy volunteer sitting on two different instances with blood flow measurement outcomes are follow using on ultrasonic blood flow sensor [2]. The device trial was carried 
out by describing gain in blood flow in the venous system of lower limb. In this work the prohibition device is synchronized with leg position and ankle of the foot, which is more practically experienced for device mobilize. The alter in deep venous blood flow were estimated by using of ultrasonic sensor measurement by principle of Doppler shift frequency.

\section{DESIGN AND ORIGIN OF METHODOLOGY}

The device is intended to assist the blood flow in a innate mechanism to improve venous discharge. The outermost system is situated outside of the muscles of the leg. The deep calf vein fundamentally receives blood flow from foot solitary [3]. Deep vein thrombosis originates in soleal vein to produce large thromboembolism which results in improper/ blockage of blood flow. The proposed model aims to imitate the mechanism of soleal pump. Flexion and extension at the ankle would result in change in area of soleus, which in turn is anticipated to change pressure with deep venous systems resulting in drain of blood similar to natural mechanism the added component of contraction is synchronized with angle fluctuation is to meant to improve the efficacy of the mechanism. The mechanism was executed by influencing a standard angle foot orthotic with only one degree of freedom.

A blood flow sensor was used to measure the velocity of blood flow and achieve high accuracy at low and high flow, save time the sensor PFMV3 is a monitor that displays the output voltage of PFMV3. A pair of accelerometer sensor is used to monitor the object movement of leg. The sensors are placed on knee and angle to provide movement on leg ADXL335 is a complete 3 -axis (x, y, z) sensing acceleration measurement system. The accelerometer can measure the static acceleration as well as dynamic resulting from movement, shock or vibration. ADXL335 is small, thin low power, complete 3 axis acceleration with signal conditioning output. Tilt sensor sense to find the position in the lower limb. The type SW-520D adopts high sensitivity and also available at affordable cost. Recent technologies in fabricate of tilt sensors have improved accuracy, reduced cost and increased lifetime. Force sensors (FSR402) is a sensor that helps in measuring the amount of force applied to an object [4].

Force sensitivity is enhanced for use in human touch control of electronic device such as locomotive electronics, medical systems and robotic application. Sensors are used wearable sock based non- invasive method diagnostic and therapeutic solutions and deep vein using lukewarm and trembling therapy by implementing lukewarm therapy, the blood flow has been improved Peltier crystal are used to provide temperature difference by transferring heat between the two electrical junction and increasing heat and function. In trembling therapy mechanical vibrator are used for movement of leg the mechanical therapy. These sensors are fed to PIC microcontroller 16F877A and the status will be updated in the MCU module and programmed in embedded $\mathrm{C}$. The device was considered to handle either in autonomous or physical mode. The autonomous mode designed for unconscious or sedated patients, whereas the manual mode is targeted towards conscious patients with confined movement. The proposed design required coexisting operation of angle variation to optimize blood flow of the leg [5].

\section{EXPERIMENTAL RESULTS}

The prototype appliance was applied on healthy subjects a ultrasonic blood flow sensor was concurrently exploit on the subject under evaluation to record any change in blood flow. The ultrasound blood flow sensor was settled upwards of the vein to estimate the blood flow in the back of the knee joint. The velocity incline displayed on the monitor was observed to gather the venue of veins and arteries [6]. The blood flow rate recorded by the device that was observed in the units of centimeters per second the device recorded blood swiftness of the chosen veins region in a buffer of limited duration [8]. The subject was asked to repose for 15 minutes in advance of the demonstration was begin. Measurement of blood flow with preventing device manipulated with lukewarm and trembling therapy at different shown in Fig 2.

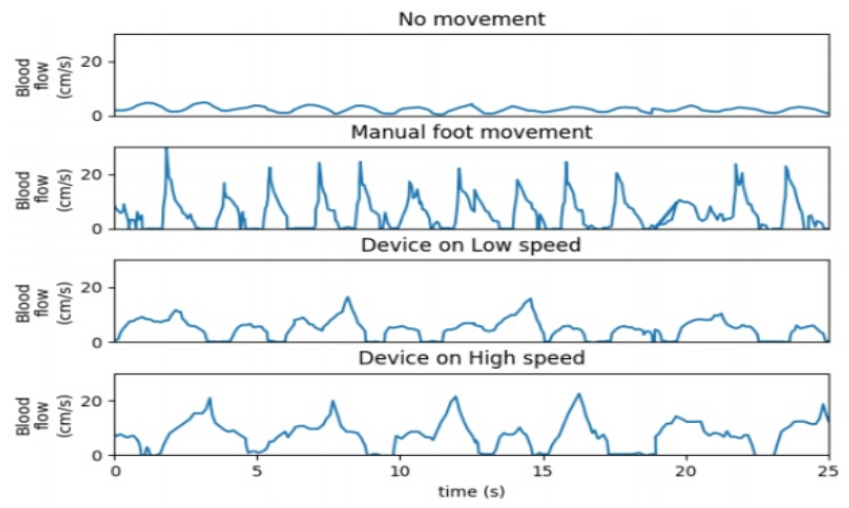

Fig 2. Measurement of blood flow with preventing device.

The tool was applied on right leg of the patient and the device was programmed to control in two different modes. These modes are high speed and low speed [7]. Table 1 indicates the blood flow rate measured by the ultrasonic blood flow 
sensor device on a patient in various condition [9]. In high-speed mode, the angle was shifted in any direction at a speed of twenty degree per second $(20 \% / \mathrm{s})$, and low speed mode actuate the angle at ten degree per second $(10 \% / \mathrm{s})$, before activating the device [8], it measures the blood flow from the subject in ultrasonic blood flow sensors without any movement and with passive angle movement [10 -12]. Blood flow rate measured by the ultrasonic blood flow sensor on a subject with different durations during treatment are inferred by Table 2 .

Table 1. Flow rate of blood

\begin{tabular}{|l|c|}
\hline Conditions & Average blood flow rate (cm/s) \\
\hline No movement & 2.16 \\
\hline Self-activating & 5.37 \\
\hline Assisted Lukewarm therapy & 4.86 \\
\hline Assisted Trembling therapy & 7.33 \\
\hline
\end{tabular}

Table 2. Flow rate of blood with different duration during treatment.

\begin{tabular}{|l|c|c|}
\hline \multirow{2}{*}{ Conditions } & \multicolumn{2}{|l|}{ Blood flow rate (cm/s) } \\
\cline { 2 - 3 } & Peak & $\begin{array}{l}\text { Average flow rate in } \\
\text { steady state }\end{array}$ \\
\hline No movement & 3.2 & 1.24 \\
\hline Assisted lukewarm therapy at slow speed & 4 & 1.22 \\
\hline Assisted lukewarm therapy at high speed & 8 & 2.44 \\
\hline Assisted trembling therapy at slow speed & 9 & 2.31 \\
\hline Assisted trembling therapy at high speed & 18 & 2.71 \\
\hline $\begin{array}{l}\text { Assisted lukewarm and trembling therapy at low } \\
\text { speed }\end{array}$ & 11 & 1.65 \\
\hline $\begin{array}{l}\text { Assisted lukewarm and trembling therapy at high } \\
\text { speed }\end{array}$ & 22 & 3.30 \\
\hline
\end{tabular}

\section{CONCLUSION}

Deep vein thrombosis is a potentially dangerous condition with a myriad of risk factors. Prophylaxis is very important and can be mechanical and pharmacological. Deep vein thrombosis therapy has become more complicated in the past few years, in fact, it has become better. Clinical manifestations like edema, tenderness, pulmonary embolus and phlegmasia cerulea doles are affected by deep vein thrombosis disease. Major risk factors for thrombosis include heart failure, pregnancy, obesity, severe respiratory disease and sepsis. Clinical diagnosis of deep vein thrombosis is unreliable because classic symptoms (pain, swelling and warmth) may not be present. Therefore, it is important for all healthcare professionals to collaborate in the identification of high-risk patient and implement prophylaxis technique. The device presented in these work attempts to mimic the natural mechanism to the fullest. The device is an example of a continuous passive leg mobility and has been modified for prophylaxis and exercise. The design is compact and is meant to serve a wide range of patients ranging from the intra-operative setting, orthopedic patients, patients suffering from neuromuscular disability and stroke. The individual mechanism via- Lukewarm and Trembling therapy have been demonstrated to improve blood flow in the leg. The two mechanisms Lukewarm and Trembling attempt to mimic the natural mechanisms which recover the blood flow. The proposed lower limb prophylaxis and exercise device is seen as a step towards developing clinically approved device, which is considered significant in the context of vast patient population having lower limb mobility issues.

\section{References}

[1]. E. Callaway, "Lukewarm reception for UK life-sciences investment," Nature, Dec. 2011.

[2]. J.H., "Lukewarm Turkey," Scientific American, vol. 260, no. 3, pp. 32-32, Mar. 1989.

[3]. Scholing and P. M. G. Emmelkamp, "Cognitive and behavioural treatments of fear of blushing, sweating or trembling," Behaviour Research and Therapy, vol. 31, no. 2, pp. 155-170, Feb. 1993.

[4]. O. S. Kerr, "A Lukewarm Defense of the Digital Millennium Copyright Act," SSRN Electronic Journal, 2002.

[5]. D. Lawrie, "Why I Am A Lukewarm Enemy Of Fundamentalism," Scriptura, vol. 99, no. 0, p. 404, Jun. 2013. 
[6]. L. Q. Kristensen, L. G. Oestergaard, K. Bovbjerg, N. Rolving, and K. Søndergaard, "Use of paraffin instead of lukewarm water prior to hand exercises had no additional effect on hand mobility in patients with systemic sclerosis: A randomized clinical trial," Hand Therapy, vol. 24, no. 1, pp. 13-21, Jan. 2019.

[7]. B. Tufty, "Our Trembling Earth," Science News, vol. 90, no. 11, p. 178, Sep. 1966.

[8]. R. W. Horst and K. Bhugra, "Method devices for moving a body joint", U. S. Patent 8353 854, Jan. 15, 2013.

[9]. T. Yonezawa, K. Nomura, T. Onodera, S. Ichimura, H. Mizoguchi, and H. Takemura, "Evaluation of venous return in lower limb by passive ankle exercise performed by PHARAD”, in Proc. 37th Annu. Int. Conf. IEEE Eng. Med. Biol. Soc. (EMBC), pp. 3582-3585, Aug. 2015.

[10]. S. M. Bögels, "Task concentration training versus applied relaxation, in combination with cognitive therapy, for social phobia patients with fear of blushing, trembling, and sweating," Behaviour Research and Therapy, vol. 44, no. 8, pp. 1199-1210, Aug. 2006.

[11]. A. Walton, R. McCrea, and R. Leonard, "Queensland survey reveals lukewarm view of coal seam gas," ECOS, 2014.

[12]. J. L. Fox, "Lukewarm reception for US biotech proposals," Nature Biotechnology, vol. 18, no. 6, pp. 590-591, Jun. 2000. 